\title{
A New Approach to Analysis of the Properties of Disordered Structures in Hydrodynamic Acoustics as Supplement to Technology and Detection Theory
}

\author{
Pavel A. Starodubtsev, Evgeny P. Starodubtsev, \\ Roman N. Alifanov and Grigory V. Dorofeev* \\ Pacific Higher Naval School \\ Vladivostok, Russian Federation
}

Received 16.03.2021, received in revised form 02.04.2021, accepted 21.05.2021

\begin{abstract}
The article presents the results of the analysis of the properties of disordered structures in hydrodynamic acoustics, associated with the process of detecting physical phenomena and marine objects based on the results of their mechanical impact on the marine environment, in which acoustic vibrations propagate. If vortices, attractors, fractals arise as a result of complex interactions of forces of nature (upwellings, seiches, Coriolis forces, currents, convection flows, rotation of the Earth) and are essentially mechanical effects on the environment of formation and propagation of an acoustic field, then mechanical sources of sound introduced into the hydrosphere (water) should repeat fractal iterations on a smaller scale at the sound field level. Recognizing the equations of hydrodynamics (the equation of motion, the equation of continuity, and the equation of state) as the fundamental equations of hydroacoustics, the nonlinearity of these equations is proposed to be considered the theory of the hydroacoustic field as nonlinear, and the linearity of the processes in this study is considered a special case. The principle of superposition also becomes a special case, and the Fourier transform, remaining necessary, loses its sufficiency. Fractal analysis in combination with wavelet analysis should be involved to help him.
\end{abstract}

Keywords: hydrodynamic acoustics, attractor, mathematical concept of a fractal, detection theory, disordered structures.

Citation: Starodubtsev P.A., Starodubtsev E.P., Alifanov R.N., Dorofeev G.V. A new approach to analysis of the properties of disordered structures in hydrodynamic acoustics as supplement to technology and detection theory, J. Sib. Fed. Univ. Eng. \& Technol., 2021, 14(3), 306-315. DOI: 10.17516/1999-494X-0310

(C) Siberian Federal University. All rights reserved

This work is licensed under a Creative Commons Attribution-Non Commercial 4.0 International License (CC BY-NC 4.0).

* Corresponding author E-mail address: spa1958@mail.ru 


\title{
Новый подход к анализу свойств \\ неупорядоченных структур \\ В гидродинамической акустике
}

как дополнение в технологию

и теорию обнаружения

\author{
П.А. Стародубцев, Е.П. Стародубцев, \\ Р. Н. Алифанов, Г. В. Дорофеев \\ Тихоокеанское высшее военно-морское училище \\ им. С. О. Макарова \\ Российская Федерация, Владивосток
}

\begin{abstract}
Аннотация. В статье представлены результаты анализа свойств неупорядоченных структур в гидродинамической акустике, связанные с процессом обнаружения физических явлений и морских объектов, по результатам их механического воздействия на морскую среду, в которой распространяются акустические колебания. Если вихри, аттракторы, фракталы возникают в результате сложных взаимодействий сил природы (апвелинги, сейши, силы Кориолиса, течения, конвекционные потоки, вращение Земли) и являются по своей сути механическими воздействиями на среду формирования и распространения акустического поля, то механические источники звука, внесенные в гидросферу (в воду), должны в более мелких масштабах повторять на уровне звукового поля фрактальные итерации. Если признать основополагающими уравнениями гидроакустики уравнения гидродинамики (уравнение движения, уравнение непрерывности и уравнение состояния), нелинейность этих уравнений вынуждает - теорию гидроакустического поля считать нелинейной, а линейность процессов в этом исследовании считать частным случаем. Принцип суперпозиции становится также частным случаем, а преобразование Фурье, оставшись необходимым, теряет свою достаточность. К нему в помощь должен быть привлечен фрактальный анализ в сочетании с вейвлет-анализом.
\end{abstract}

Ключевые слова: гидродинамическая акустика, аттрактор, математическое понятие фрактала, теория обнаружения, неупорядоченные структуры.

Цитирование: Стародубцев, П. А. Новый подход к анализу свойств неупорядоченных структур в гидродинамической акустике как дополнение в технологию и теорию обнаружения / П. А. Стародубцев, Е. П. Стародубцев, Р.Н. Алифанов, Г. В. Дорофеев // Журн. Сиб. федер. ун-та. Техника и технологии, 2021, 14(3). С. 306-315. DOI: 10.17516/1999-494X-0310

\section{Введение}

Современные технологии формирования систем обнаружения и регистрации сигналов в первую очередь связаны с совершенствованием процесса передачи и приема информации об объектах, находящихся в водной среде, их классификационными или распознавательными характеристиками. При этом распознавательные характеристики объектов определяются субъективным принципом принятия решения, т. е. ограничены понятием «субъективной классификации», где вторичные признаки считаются основными без учета «физического» объяснения причин их возникновения. Факт обнаружения является результатом анализа информации по плоским первичным волнам, а получение «вторичных признаков» - по итогам их синтеза на апертуре акустической антенны. 
Научная задача исследований будет связана с процессом аналитического рассмотрения не систем, формирующих гидроакустические сигналы или их принимающие, а среды, в которой распространяются акустические колебания, т. е. воды и ее «неупорядоченных» систем, в которых отсутствует дальний порядок в расположении частиц. При этом к неупорядоченным системам отнесем жидкости, аморфные и стекловидные вещества. Несмотря на отсутствие дальнего порядка, ближний порядок в таких системах может сохраняться.

\section{Основная часть}

При проведении научно-исследовательских работ (НИР) в период с 2008 по 2017 г. в заливе Стрелок у северо-восточного берега залива Петра Великого Японского моря (южное побережье Приморского края), в отдельных районах у острова Путятин, лежащего посередине залива и делящего его почти пополам, были обнаружены неупорядоченные системы (вихревые аномалии), обладающие свойствами странных аттракторов, переходящих во фрактальные области. При изменении их пространственных размеров эти аномалии сохраняли свои геометрические и физические свойства самоподобия от итерации к итерации [1] (рис. 1).

По своей структуре залив Стрелок напоминает естественный резонатор, в котором возможно внутреннее переотражение акустических волн, практически без потери энергии.

Динамика формы аттрактора постоянно в различных масштабах монотонно убывала, затем возрастала и проявляла фрактальное самоподобие. Эти факты дали возможность пояснить причины, послужившие основанием для обнаружения звуковых вариаций самого аттрактора и шумового поля движущегося морского объекта без визуального его наблюдения на поверхности, а интеграция аттрактора и его динамики в виде фрактала явилась условием обнаружения акустического поля от объектов естественного и искусственного происхождения в водной среде в условиях материковой отмели и закрытых бухт [1].

Такой же звук наблюдался и фиксировался и от банки Бонсдорфа (банка у бухты Нерпичья, западная часть залива Петра Великого) (рис. 2) и представлял собой шумовой фрактал, по структуре подобный вихревым и турбулентным шумам.

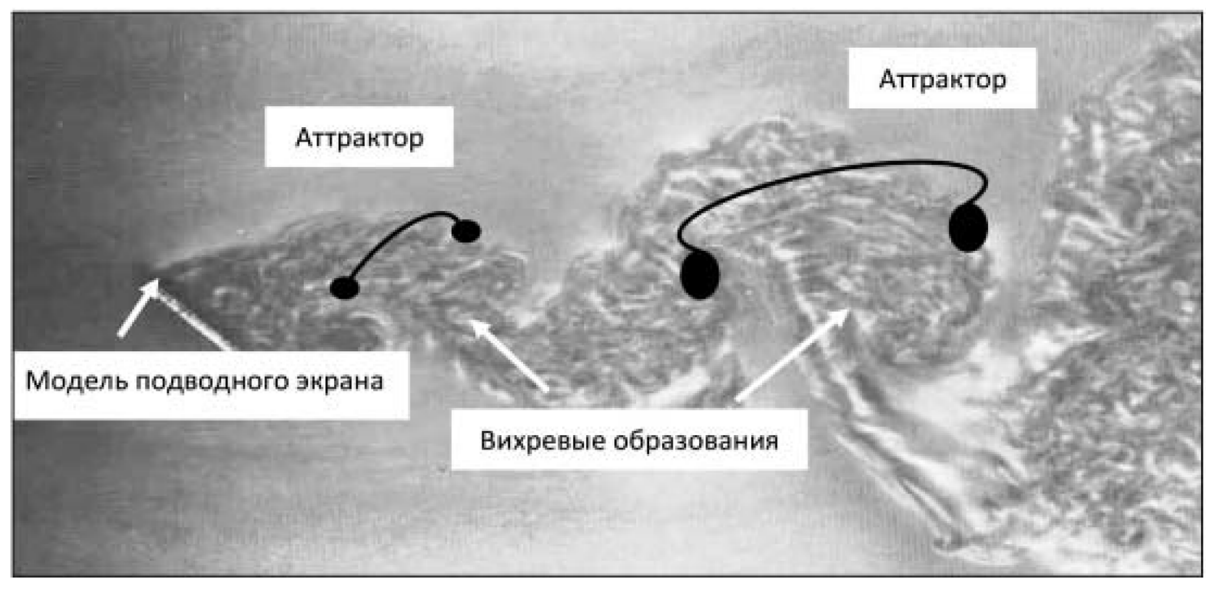

Рис. 1. Странные аттракторы, переходящие во фрактальные области [1]

Fig. 1. Strange attractors turning into fractal regions [1]

$$
-308-
$$




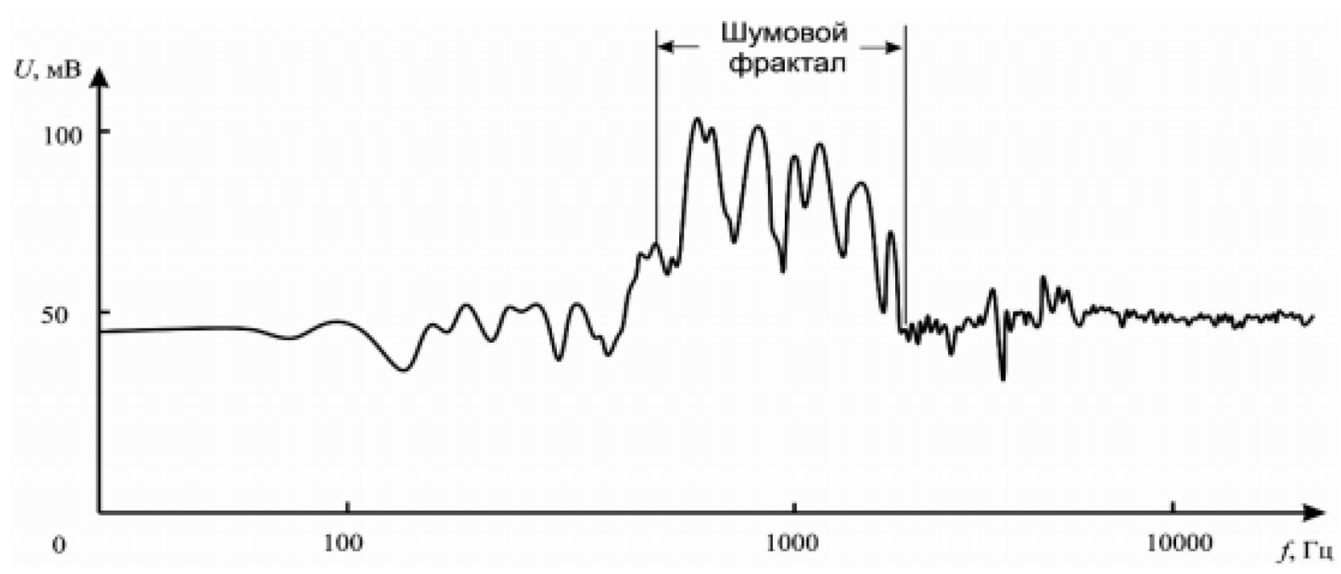

Рис. 2. Шумы от банки Бонсдорфа [1]

Fig. 2. Noises from the Bonsdorf bank [1]

Причиной появления такого физического «самоподобия», по мнению авторов, служит географическая структура залива Стрелок. Он вдается в сушу между мысом Майделя и мысом Гембачева, длина его около 12 км, ширина до 15 км, глубина до 46 м. Берега залива высокие и каменистые. В берега залива вдается несколько бухт, крупнейшие из которых Разбойник, Абрек, Руднева, Чажма, а также бухта Назимова на острове Путятина. Кроме острова Путятина также имеется еще несколько более мелких островков и скал: остров Никольского, камни Унковского, остров Ирецкого, кекуры Пять Пальцев.

Данные научные выводы (частично) в 2014 г. также подтвердили сотрудники [2] Государственного океанографического института им. Н.Н. Зубова и Санкт-Петербургского государственного университета (А.Е. Зверева и В.Р. Фукс). Они выдвинули гипотезу о наличии в Японском море «котловинных» волн. По мнению авторов статьи, свойства «котловинных» волн близки «самоподобным» вихревым потокам в мелководных районах залива Петра Великого (рис. 3).

С точки зрения А.Е. Зверева и В. Р. Фукс [2], есть основания полагать, что в котловине Уллын Японского моря происходит топографический захват энергии этих волн. «Котловинные» волны принадлежат классу градиентно-вихревых волн, они обязаны своим происхождением совместному эффекту вращения и сферичности Земли, а также топографическим особенностям акватории. «Котловинные» волны, наряду с шельфовыми, склоновыми и «желобовыми» (двойными волнами Кельвина), можно также отнести к классу топографических волн Россби.

Для выяснения особенностей динамики топографических волн [2] были подготовлены массивы альтиметрических данных с 1992 по 2013 г. и оценены статистические характеристики изменчивости альтиметрического рельефа поверхности моря при различных масштабах пространственно-временного осреднения. Подтвердилось предположение о доминировании стояче-поступательных волн, что позволяет объяснить многие ранее не объяснявшиеся явления в Японском море, такие как скачкообразное изменение фазы при перемещении неоднородностей в поле уровня, ячеистая структура возмущений уровня и течений с выраженными амфидромиями в центрах этих ячеек [2].

$$
-309-
$$




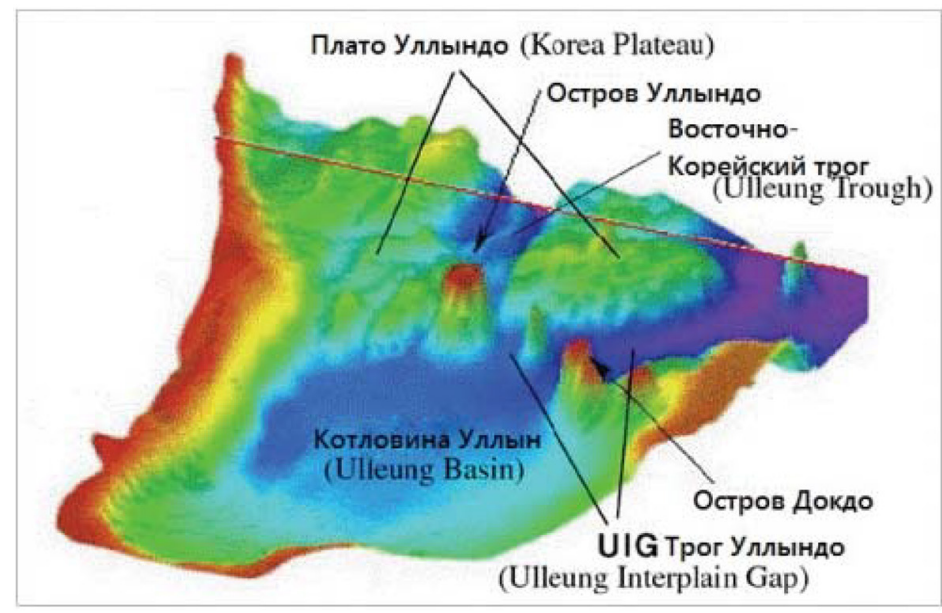

Рис. 3. Донная топография котловины Уллын (Японское море) [2]

Fig. 3. Bottom topography of the Ulleung Basin (Sea of Japan) [2]

Было отмечено, что увеличение кинетической энергии колебаний в котловине свидетельствует о том, что существует физический механизм, аккумулирующий эту энергию в котловине [2].

В пространственных распределениях амплитуд и начальных фаз четко выделяются области с пониженными значениями амплитуд и существенным сгущением линий изофаз (рис. 4). Согласно кинематике стоячих и стоячепоступательных волн в теории приливов данные области являются амфидромическими. Годовой период отличается повышенной амплитудой уровня моря в области теплого вихря Уллын на северо-западе области с относительно низкими значениями на юго-востоке, где отмечается холодный вихрь Док. Эти две зоны окружены кольцевыми котидальными линиями. Полугодовой период имеет обратную картину распределения максимумов: положительная аномалия амплитуды находится юго-восточнее отрицательной [2] (рис. 4).

Логика анализа представленных выше данных $[1,2]$ привела к выводу, что самоподобие (как физическое явление) должно отмечаться и в других условиях. Достаточно «сложное» для полного объяснения влияние на структуру и характер акустического поля среды (например, в бухте Стрелок залива Петра Великого) оказывают сейши. Как показали исследования [3], в данном районе они представляют собой [3] стоячие свободные колебания жидкости в «полузамкнутом» пространстве (рис. 5) с формированием одноузловой структуры.

Если упомянутые выше неупорядоченные системы возникают [1-3] в результате сложных взаимодействий сил природы (апвелинги, сейши, силы Кориолиса, течения, конвекционные потоки, вращение Земли) и являются по своей сути механическими воздействиями на среду формирования и распространения акустического поля, то механические источники звука, внесенные в гидросферу (в воду), должны в более мелких масштабах повторять на уровне звукового поля фрактальные итерации.

\section{Решение научной задачи}

Нужно только знать, какими размерами колеблющихся частиц следует оперировать в этом случае. Что значит выражение: «В гидродинамике среда считается сплошной и рассматрива- 


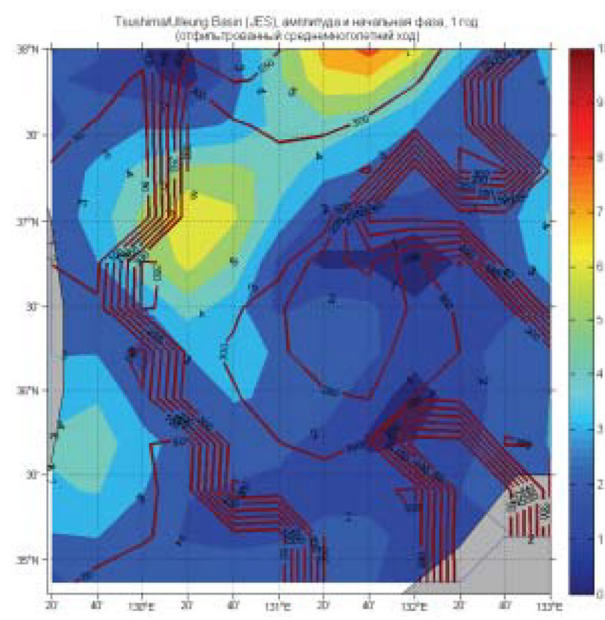

a)

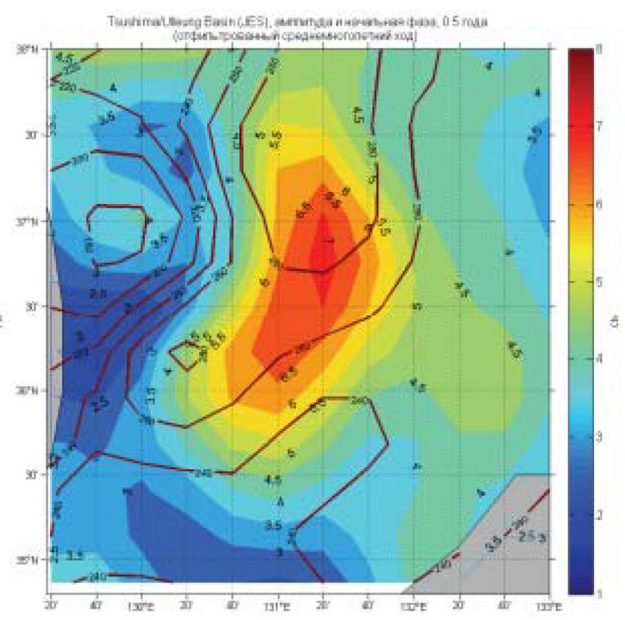

б)

Рис. 4. Карты амплитуд и начальных фаз аномалий уровня для годового (а) и полугодового (б) периода [2]

Fig. 4. Maps of amplitudes and initial phases of level anomalies for annual (a) and semi-annual (6) periods [2]

a)

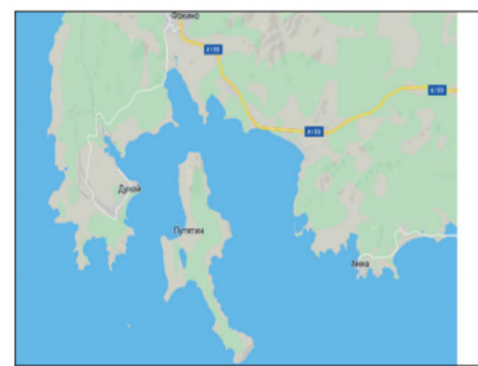

б)

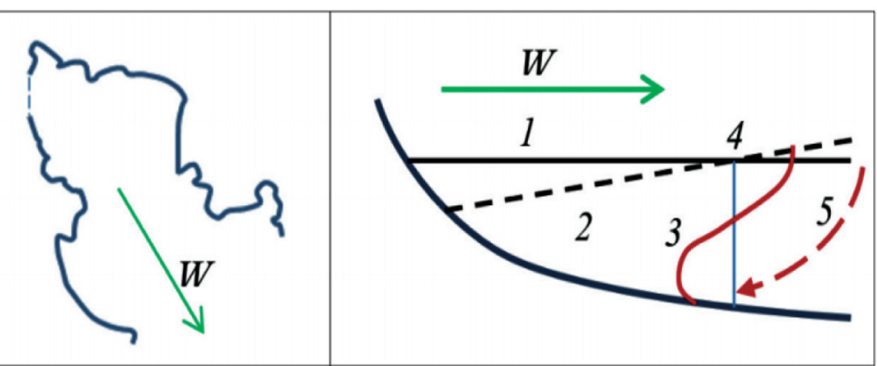

Рис. 5. Формирование одноузловой сейши: а - акватория залива Петра Великого; б - направление нагонного ветра; в - последовательность появления одноузловой сейши [3]

Fig. 5. Formation of a one-node seiche: a - water area of the Peter the Great Bay; 6 - the direction of the surge wind; $\mathrm{B}$ - the sequence of the appearance of a single-node seiche [3]

емый в ней объем среды мал в сравнении с размерами макроскопических тел, но достаточно велик по сравнению с расстоянием между молекулами» [4-5]? Это сколько?

Если мы беремся решать задачу обнаружения акустически малозаметных объектов, то прежде всего необходимо пересмотреть в теории гидроакустики целый ряд положений, содержащих ранее принятые допущения и линеаризации. Проанализировать их справедливость на этапе более высокого уровня математического аппарата обработки потоков информации вообще и акустических исследований в частности. Согласиться с тем, что теория гидроакустического поля имеет свои совершенно специфические особенности, не дающие возможность использовать аппарат исследования, пригодный для исследования газов и твердых тел.

Нельзя полностью переносить теорию волновых процессов в атмосфере на волновые процессы в морской среде. Примером может служить изотопный фактор строения воды. Физический энциклопедический словарь [6] трактует, что вследствие существования 3 изотопов водо- 
рода - протия, дейтерия и трития - и 6 изотопов кислорода - $O^{14}, O^{15}, O^{16}, O^{17}, O^{18}, O^{19}$ имеется 36 изотопных разновидностей воды.

Если признать основополагающими уравнениями гидроакустики уравнения гидродинамики (уравнение движения, уравнение непрерывности и уравнение состояния), нелинейность этих уравнений вынуждает считать теорию гидроакустического поля нелинейной, а линейность процессов в этом исследовании - частным случаем.

Признание этого фактора требует пересмотра некоторых позиций, о которых будет сказано ниже. Сейчас отметим только то, что принцип суперпозицим становится также частным случаем, а преобразование Фурье, оставшись необходимым, теряет свою достаточность. К нему в помощь должен быть привлечен фрактальный анализ в сочетании с вейвлет-анализом. Это, в свою очередь, неизбежно приведет к пространственному разложению функции, т. е. к тензорному аппарату вычислений [7].

\section{Концептуальный пересмотр теории акустического поля}

Это должно стать концептуальным пересмотром теории акустического поля для условий обнаружения акустически малозаметных объектов.

Акустические процессы в водной среде относятся к процессам, в которых микроскопические связи между частицами среды определяют макроскопическое поведение всей среды.

Природа устроена таким образом, что микроскопическая структура многих неупорядоченных объектов обладает свойством масштабной инвариантности (скейлинга). Масштабная инвариантность позволяет строить теорию таких сред с помощью методов ренормализационной группы (также часто называемый методом ренормгруппы, методом РГ).

В квантовой теории поля это итеративный метод перенормировки, в котором переход от областей с меньшей энергией к областям с большей вызван изменением масштаба рассмотрения системы.

\section{Модель нетривиальной масштабно-инвариантной структуры}

Этот подход универсален, но связан с объемными вычислительными процессами, которые еще десять лет назад были трудно осуществимы. Развитое Мандельбротом математическое понятие фрактала (по Мандельброту, изломанный объект с дробной размерностью) и его приложения к описанию форм различных объектов дают возможность составить модель нетривиальной масштабно-инвариантной структуры, похожей на кластерную сруктуру «слияния» частиц водной среды $[8,9]$.

\section{Новый подход к анализу неупорядоченных структур в гидродинамической акустике}

В применении такой модели заключается новый подход к анализу неупорядоченных структур в гидродинамической акустике. Фрактальные модели не всегда поддаются аналитическому исследованию, но могут быть построены по простым правилам с возможностью несложной компьютерной реализации. С помощью языка программирования императивного типа со строгой статической типизацией переменных «Delphi» можно строить фракталы 
самых разнообразных форм. Закономерности сложных неупорядоченных процессов изучают в компьютерных экспериментах с подобными моделями.

Такой подход значительно отличается от традиционных методов теоретической физики (например, выбор точек обработки информации при анализе сигнала по уравнению Бине и его частному случаю - числу Фибаноччи). Это отличие не сводится к разнице между численным решением дифференциальных уравнений и их аналитическим исследованием.

\section{Фракталы как нелинейные процессы}

Фракталы позволяют продвинуться в исследовании акустических процессов как нелинейных процессов. Причем под нелинейностью понимается не частный случай, обусловленный энергетическими всплесками, а нормальное состояние среды, в которой формируется и распространяется акустическое поле, среды, в которой линейные процессы являются идеализированными случаями теоретического плана.

Все акустические процессы в водной среде должны изначально приниматься к рассмотрению с позиций нелинейности. Должны рассматриваться условия согласованности источника колебаний в водной среде со свойствами самой водной среды.

Поясним это примером. Принимаем, что однородная водная среда имеет постоянный коэффициент упругости $k_{\text {упр }}$, благодаря которому при восстановлении состояния среды после адиабатического сжатия возврат частиц в исходное состояние происходит со скоростью $v_{\text {упр }}$.

Источник звуковых волн, внесенный в водную среду, вибрирует с такой частотой, что вы-

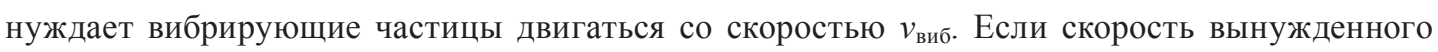
движения равна собственной скорости движения частиц воды за счет собственных свойств среды, обусловленных упругими ее свойствами, т. е. имеет место равенство

$$
v_{\text {упр }}=v_{\text {виб̆ }}
$$

то можно говорить о линейности процессов, происходящих в среде.

При уменьшении частоты колебаний источника наступает фаза неравенства скоростей типа $v_{\text {упр }}>v_{\text {виб. }}$ В этом случае частицы воды будут обгонять частицы поверхности излучателя, искажать гармоничность колебательного процесса и создавать дополнительные напряжения между излучающей поверхностью и водной средой, которые приводят к «измельчению» кластерной структуры водной среды в зоне колебания.

При увеличении частоты колебаний источника наступает фаза типа $v_{\text {упр }}<v_{\text {виб. }}$ В этом случае частицы воды будут отставать от частиц поверхности излучателя, искажая гармоничность колебания среды, и создавать «пустоты» в среде, неизбежное сжатие которых вызывает микровзрывы и разрушение кластерной структуры воды.

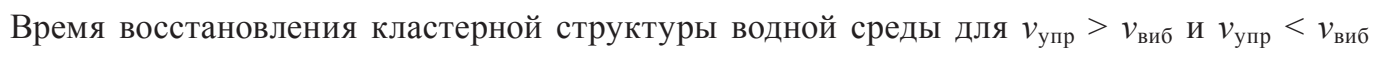
случаев неравенства скоростей будет определяться в общем случае различными алгоритмами. Время восстановления кластерной структуры будет иметь разную величину.

\section{Заключение}

В итоге если время восстановления кластерной структуры воды составляет $\mathrm{t}_{\text {вост }}$, то за про-

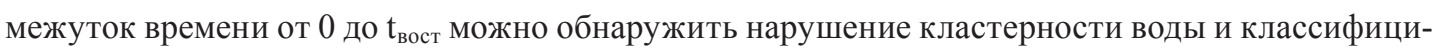


ровать по частоте источник излучения (частота излучения выше собственных колебаний среды или ниже). Дополнительную окраску классификационных признаков источников колебаний дает смачиваемость вибрирующей поверхности, приводящей к дополнительному искажению гармоничности его колебания. Становится классификационным признаком обрастание биологическими организмами вибрирующих поверхностей. Аномально высокая теплоемкость воды обеспечивается многочисленными водородными связями (механизм Гротгуса). Одна молекула может образовать до четырех классических водородных связей с соседями (с учетом Н-связей до 5-6). Водородные связи повышают температуру кипения, вязкость и поверхностное натяжение жидкости, они ответственны за многие другие уникальные свойства воды. Фрактальность водной среды определяется соленостью, температурой, плотностью и давлением. Исследование фрактальности воды неизбежно повлечет за собой изменение содержания эмпирических формул расчета скорости звука в воде.

Представленные в работе выводы могут быть реализованы новой технологией системы регистрации акустически слабозаметных объектов по первичным признакам гидродинамических характеристик среды и шумовых полей объектов с использованием приборов и устройств векторного анализа и синтеза.

\section{Список литературы / References}

[1] Стародубцев П.А., Халаев Н.Л., Бакланов Е.Н. Характеристики гидродинамических портретов акустических полей мелководных районов залива Петра Великого Японского моря. Вестник Государственного университета морского и речного флота имени адмирала С. О. Макарова, 2019, 11(1), 87-98. [Starodubtsev P.A., Khalaev N.L., Baklanov E.N. Characteristics of hydrodynamic portraits of acoustic fields in shallow water areas of Peter the Great Bay, Sea of Japan. Bulletin of the State University of Marine and River Fleet named after Admiral S. O. Makarov, 2019, 11(1), 87-98 (in Russian)]

[2] Зверева А.Е., Фукс В.Р. Градиентно-вихревые волны в котловине Уллын Японского моря. Современные проблемы дистанционного зондирования Земли из космоса, 2014, 11(3), 1927. [Zvereva A. E., Fuks V. R. Gradient vortex waves in the Ulleung Basin of the Sea of Japan. Modern problems of remote sensing of the Earth from space, 2014, 11(3), 19-27 (in Russian)]

[3] Стародубцев П. А., Халаев Н. Л., Бакланов Е.Н. Исследование процесса влияния сейши на акустическое поле замкнутых акваторий мелководных районов. Вестник Государственного университета морского и речного флота имени адмирала С. О. Макарова, 2018, 10(1), 183-190. [Starodubtsev P. A., Khalaev N.L., Baklanov E. N. Investigation of the process of seiche influence on the acoustic field of closed water areas of shallow water areas, Bulletin of the State University of Marine and River Fleet named after Admiral S. O. Makarov, 2018, 10(1), 183-190 (in Russian)]

[4] Мироненко М.В., Малашенко А.Е., Василенко А.М. [и др.]. Нелинейная просветная гидроакустика и средства морского приборостроения в создании дальневосточной радиогидроакустической системы освещения атмосферы, океана и земной коры, мониторинга их полей различной физической природы. Владивосток: ФГБУН СКБ САМИ ДВО РАН, 2014. 402 c. [Mironenko M.V., Malashenko A.E., Vasilenko A.M. [and etc.]. Nonlinear transmissive hydroacoustics and marine instrumentation in the creation of a Far Eastern radio-hydroacoustic 
system for lighting the atmosphere, ocean and earth's crust, monitoring their fields of various physical nature. Vladivostok, FGBUN SKB SAMI FEB RAS, 2014. 402 p. (in Russian)]

[5] Халаев Н.Л., Стародубцев П.А., Шевченко А.П. Фрактальное моделирование в гидродинамической акустике. Всероссийская 56-я научная конференщия «Фундаментальные и прикладные вопросы естествознания». Владивосток: филиал ВУНЦ ВМФ «ВМА», 2013, 3, 285-289. [Khalaev N.L., Starodubtsev P.A., Shevchenko A.P. Fractal modeling in hydrodynamic acoustics. All-Russian 56th Scientific Conference «Fundamental and Applied Questions of Natural Science». Vladivostok: branch of the VUNC Navy «VMA», 2013, 3, 285-289 (in Russian)]

[6] Введенский Б.А. Физический энщиклопедический словарь, М.: Наука, 1963-1968. [Vvedensky B. A. Physical encyclopedic dictionary, Moscow, Nauka, 1963-1968 (in Russian)]

[7] Горовой С.В. Некоторые результаты экспериментального оценивания трехмерных плотностей распределения выборочных значений давления гидроакустических шумов в заливе Петра Великого Японского моря. VIII Всероссийского симпозиум «Физика геосфер». Владивосток, ТОИ ДВО РАН, 2013, 59-63. [Gorovoy S. V. Some results of experimental estimation of three-dimensional distribution densities of sample values of hydroacoustic noise pressure in the Peter the Great Bay of the Sea of Japan. VIII All-Russian Symposium «Physics of Geospheres». Vladivostok, POI FEB RAS, 2013, 59-63 (in Russian)]

[8] Шустиков А.Г. К вопросу о динамических связях пульсаций давления с полем скорости внутри турбулентного потока при малых числах Маха. Акустический журнал, 1983, 29(5), 693-699. [Shustikov A.G. On the question of dynamic relationships between pressure fluctuations and the velocity field inside a turbulent flow at low Mach numbers. Acoustic magazine, 1983, 29(5), 693-699 (in Russian)]

[9] Мироненко М.В., Василенко А. М., Карачун Л.Э. Информационно-аналитическая система расчета и анализа просветного гидроакустического поля в системах мониторинга морских акваторий, Датчики и системь, 2015, 9-10(196), 12-16. [Mironenko M. V., Vasilenko A. М., Karachun L.E. Information-analytical system for calculating and analyzing the translucent hydroacoustic field in systems for monitoring sea areas, Sensors and systems, 2015, 9-10(196), 12-16 (in Russian)] 